\title{
The role of watermelon caffeic acid O-methyltransferase (CICOMT1) in melatonin biosynthesis and abiotic stress tolerance
}

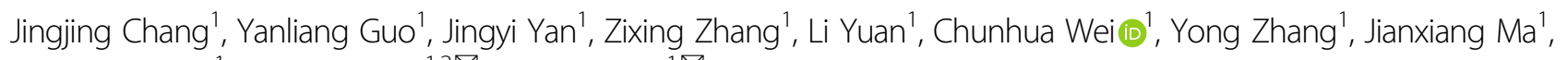
Jianqiang Yang ${ }^{1}$, Xian Zhang $\mathbb{B}^{1,2 \otimes}$ and HaO Li (iD)

\begin{abstract}
Melatonin is a pleiotropic signaling molecule that regulates plant growth and responses to various abiotic stresses. The last step of melatonin synthesis in plants can be catalyzed by caffeic acid O-methyltransferase (COMT), a multifunctional enzyme reported to have $\mathrm{N}$-acetylserotonin O-methyltransferase (ASMT) activity; however, the ASMT activity of COMT has not yet been characterized in nonmodel plants such as watermelon (Citrullus lanatus). Here, a total of 16 putative O-methyltransferase (CIOMT) genes were identified in watermelon. Among them, CIOMT03 (Cla97C07G 144540) was considered a potential COMT gene (renamed CICOMT1) based on its high identities (60.00-74.93\%) to known COMT genes involved in melatonin biosynthesis, expression in almost all tissues, and upregulation under abiotic stresses. The CICOMT1 protein was localized in the cytoplasm. Overexpression of CICOMT1 significantly increased melatonin contents, while CICOMT1 knockout using the CRISPR/Cas-9 system decreased melatonin contents in watermelon calli. These results suggest that CICOMT1 plays an essential role in melatonin biosynthesis in watermelon. In addition, CICOMT1 expression in watermelon was upregulated by cold, drought, and salt stress, accompanied by increases in melatonin contents. Overexpression of CICOMT1 enhanced transgenic Arabidopsis tolerance against such abiotic stresses, indicating that CICOMT1 is a positive regulator of plant tolerance to abiotic stresses.
\end{abstract}

\section{Introduction}

Melatonin ( $N$-acetyl-5-methoxytryptamine), an important bioactive molecule, is ubiquitously present in living organisms throughout the animal, plant, and many other kingdoms ${ }^{1,2}$. Melatonin was identified in vascular plants in $1995 ;{ }^{3,4}$ since then, numerous studies have proven the multiple functions of melatonin in plant growth and development, postharvest physiology, and adaptation to various environmental stresses, such as cold, salinity, drought, and pathogens ${ }^{5-7}$. Recently, the discovery of the first melatonin receptor (CAND2/PMTR1) in Arabidopsis

\footnotetext{
Correspondence: Xian Zhang (zhangxian@nwafu.edu.cn) or Hao Li (yuanyilihao123@163.com)

'State Key Laboratory of Crop Stress Biology for Arid Areas, College of Horticulture, Northwest A\&F University, Yangling, Shaanxi, China

${ }^{2}$ State Key Laboratory of Vegetable Germplasm Innovation, Tianjin, China
}

provided strong evidence for considering melatonin as a new plant hormone $\mathrm{e}^{8,9}$.

The quantities of melatonin in plant cells vary considerably among species, ranging from several picograms to a few micrograms per gram of fresh tissue ${ }^{10}$. Melatonin in plants is synthesized from tryptophan via four consecutive enzymatic steps ${ }^{6,11,12}$. In the first two steps, tryptophan is converted into serotonin by tryptophan decarboxylase, tryptophan hydroxylase, and tryptamine 5-hydroxylase. In the final two steps, serotonin is catalyzed into $\mathrm{N}$-acetylserotonin (NAS) by serotonin $\mathrm{N}$-acetyltransferase (SNAT) in chloroplasts, and NAS is subsequently methylated into melatonin by $N$-acetylserotonin $O$-methyltransferase (ASMT) in the cytoplasm ${ }^{13}$. Some studies suggest that ASMT, as the terminal enzyme, plays a rate-limiting role in melatonin biosynthesis in plants ${ }^{6,14}$.

\section{(c) The Author(s) 2021}

(c) (i) Open Access This article is licensed under a Creative Commons Attribution 4.0 International License, which permits use, sharing, adaptation, distribution and reproduction cc) in any medium or format, as long as you give appropriate credit to the original author(s) and the source, provide a link to the Creative Commons license, and indicate if changes were made. The images or other third party material in this article are included in the article's Creative Commons license, unless indicated otherwise in a credit line to the material. If material is not included in the article's Creative Commons license and your intended use is not permitted by statutory regulation or exceeds the permitted use, you will need to obtain permission directly from the copyright holder. To view a copy of this license, visit http://creativecommons.org/licenses/by/4.0/. 
The first $A S M T$ gene (OsASMT1) was cloned from the monocotyledonous species rice (Oryza sativa) in $2011^{15}$. In addition to OsASMT1, overexpression of OsASMT2 and OsASMT3 in rice calli also enhanced melatonin production ${ }^{16}$. Subsequent studies cloned the $A S M T$ genes from dicotyledonous plant species, including Arabidopsis, Malus zumi, and Hypericum perforatum $^{17-19}$. However, the homologies of ASMT genes vary considerably among plant species. The homologous genes of rice $A S M T$ are found only in some other monocotyledonous plants ${ }^{15,16}$. ASMT genes from dicotyledonous Arabidopsis and Malus zumi encoded proteins that share only $31 \%$ and $40 \%$ identity, respectively, with OsASMT1 $1^{17,18}$. In addition to ASMT, caffeic acid $O$-methyltransferase (COMT), a multifunctional enzyme, was also reported to have ASMT activity ${ }^{20,21}$. Based on enzyme catalysis, both ASMT and COMT belong to the $O$-methyltransferase (OMT) family, which methylates a variety of secondary metabolites, such as phenylpropanoids, alkaloids, and flavonoids ${ }^{20,22}$. COMT genes have been identified in various plant species, such as Lolium perenne, Chrysanthemum grandiflorum, Hordeum vulgare, Brachypodium distachyon, and Populus tremuloides ${ }^{23-29}$. However, their involvement in melatonin biosynthesis was reported only in model plants, including monocotyledonous rice and dicotyledonous Arabidopsis and Solanum lycopersicum $^{20,21,30}$. Melatonin biosynthesis-related COMT genes from other plant species need to be further identified to exploit their new functions. Moreover, COMT genes with ASMT function from crops can be utilized for enhancement of crop yield and quality through genetic manipulation and rational breeding.

Watermelon (Citrullus lanatus (Thunb.) Matsum. \& Nakai), one of the top five most consumed fresh fruits, is widely grown as an important horticultural crop worldwide. The cultivated area of watermelon in 2018 was 3.24 million hectares worldwide (http://www.fao. org/). Previous investigators have reported the positive effects of exogenous melatonin on watermelon tolerance against abiotic stresses, including cold, salt, drought, and vanadium stress; ${ }^{31-34}$ however, the melatonin biosynthetic genes in watermelon have not been cloned and characterized due to the relatively difficult process of genetic transformation in watermelon. Here, we performed bioinformatics analysis of watermelon ClOMT genes, cloned a potential COMT gene (renamed ClCOMT1), and further characterized its function in melatonin production via overexpression or knockout of ClCOMT1 in watermelon calli. In addition, we investigated the role of ClCOMT1 in tolerance against abiotic stresses, including cold, drought, and salinity, by overexpressing ClCOMT1 in Arabidopsis.

\section{Materials and methods}

\section{Identification of O-methyltransferase (OMT) genes in watermelon}

The watermelon protein database (watermelon_v2.pep) was obtained from the Cucurbit Genomics Database (CuGenDB, http://cucurbitgenomics.org/) ${ }^{35}$. Then, the hidden markov model (HMM) profile of the O-methyltransferase domain (PF00891) from Pfam (http://pfam. xfam.org/) was utilized to identify OMT genes from the watermelon protein database with an e-value $<1 \times \mathrm{e}^{-10}$. The presence of the PF00891 domain in all the protein sequences obtained was analyzed using the SMART tool (http://smart.embl-heidelberg.de/) ${ }^{36}$.

\section{Chromosomal locations and phylogenetic analysis}

The chromosomal location image of ClOMTs was drawn using MapChart $2.32^{37}$, on the basis of chromosomal position information from the watermelon genome website (http://cucurbitgenomics.org/organism/ 21). Tandem duplicated genes were identified based on two criteria: (a) adjacent homologous genes were located on the same chromosome with no more than one intervening gene, and (b) the length and similarity of aligned sequences were $>70 \%{ }^{38,39}$. Multiple sequence alignments of ClOMT protein sequences were carried out via ClustalW with default parameters ${ }^{40}$. Based on the alignment results, a phylogenetic tree was constructed in MEGA 7.0.21 software by using the neighbor-joining method with 1000 bootstrap replications and a Jones-Taylor-Thornton (JTT) matrix-based $\operatorname{model}^{41}$.

\section{Expression analysis of CIOMT genes based on mRNA sequencing}

The expression of watermelon $C l O M T$ genes in different tissues and in response to abiotic stresses was analyzed based on mRNA sequencing data from CuGenDB (http://cucurbitgenomics.org/). The tested tissues included fruit flesh, fruit rind 10, 18, 26, and 34 days after pollination, seeds 49 days after pollination, phloem, vascular bundle, root, and leaf. For abiotic stress treatments, the watermelon seedlings were exposed to $4{ }^{\circ} \mathrm{C}$ for $6 \mathrm{~h}$, water was withheld for eight days, or PEG 6000 (20\%) was applied to the roots for $6 \mathrm{~h}$. Leaf tissues were harvested after cold and drought, while root samples were taken after PEG 6000 treatment. Transcript levels were calculated as reads per kilobase of exon model per million mapped reads (RPKM). The RPKM values were $\log 2$ transformed for tissue-specific analysis, while the RPKM ratio of Treatment/Control was $\log 2$ transformed for stress response analysis. The heat maps were drawn using Multiple Experiment Viewer (Version Mev4.9) ${ }^{42}$. 


\section{Subcellular localization of CICOMT1}

The pGREEN vector fused with green fluorescent protein (GFP) was used to determine the subcellular localization of the ClCOMT1 protein. The cDNA sequence of ClCOMT1 was PCR-amplified with primers containing $E c o R V$ and $X h o I$ restriction sites. The amplified products were gel-purified and ligated into the pGREEN vector. Then, the recombinant plasmid pGREEN-ClCOMT1-GFP was transiently transformed into watermelon leaf protoplasts. Protoplast extraction and transfection were performed as described previously using 20-day-old watermelon leaves ${ }^{43}$. Fluorescence was observed using a confocal laser scanning microscope (Leica TCS-SP8 SR, Germany).

\section{Vector construction and gene transformation in watermelon calli}

To knock out ClCOMT1 in wild-type watermelon calli, the CRISPR/Cas9 construct was generated using the binary vector PBSE402 with two guide RNAs designed by CRISPR-P v2.0 (http://crispr.hzau.edu.cn/CRISPR2/) ${ }^{44,45}$. Overexpression of ClCOMT1 in watermelon calli was completed using the pCambia1305.4 vector. A 1074-bp ClCOMT1 cDNA was PCR-amplified with specific primers harboring BamHI and PmlI restriction sites and was then ligated into the pCambia1305.4 vector. Recombinant PBSE402-ClCOMT1 or pCambia1305.4-ClCOMT1 was transformed into watermelon by Agrobacterium tumefaciens-mediated transformation (strain EHA105) ${ }^{43}$. A potentially successful transformation was detected by monitoring GFP fluorescence under a stereoscopic fluorescence microscope (MZ10F, Leica, Germany). Genomic DNA fragments with target sites were amplified to determine the mutation types using high-throughput tracking of mutations $(\mathrm{Hi}-\mathrm{TOM})^{46}$. Expression of ClCOMT1 in the transgenic or wild-type calli was analyzed by qRT-PCR. Specific amplification primers are given in Supplementary Table S1.

\section{Transgenic Arabidopsis plant generation and abiotic stress tolerance assay}

To overexpress ClCOMT1 in Arabidopsis, the recombinant plasmid pGREEN-ClCOMT1-GFP was transformed into Agrobacterium tumefaciens (strain GV3101), which was then transformed into Arabidopsis using the floral dip method ${ }^{47}$. Transgenic Arabidopsis plants were screened by BASTA. Transcripts of ClCOMT1 were determined in the leaves of four-week-old wild-type or $\mathrm{T}_{0}$ transgenic Arabidopsis using semiquantitative RT-PCR. Detailed primer sequences are given in Supplementary Table S1.

Homozygous $\mathrm{T}_{3}$ transgenic line \#4 was used for analysis of abiotic stress tolerance. After vernalization at $4{ }^{\circ} \mathrm{C}$ for $3 \mathrm{~d}$, transgenic and wild-type Arabidopsis seeds were cultured on half-strength Murashige and Skoog (MS) plates in a growth chamber at $22^{\circ} \mathrm{C}$. Freezing stress treatment was carried out as described by $\mathrm{Hu}$ et al. with a slight modification ${ }^{48}$. Two-week-old seedlings were exposed to $-10^{\circ} \mathrm{C}$ for $1 \mathrm{~h}$ and recovered at $22^{\circ} \mathrm{C}$ for $4 \mathrm{~d}$. For drought and salt stress treatments, the vernalized seeds were sown and grown in $1 / 2$ MS containing $250 \mathrm{mM}$ D-mannitol and $75 \mathrm{mM} \mathrm{NaCl}$ for 18 days, respectively $^{18,49}$. Survival rates of Arabidopsis seedlings were recorded.

\section{Quantitative RT-PCR and semiquantitative RT-PCR}

Total RNA was extracted using the RNA simple Total RNA Kit (TIANGEN, Beijing, China) following the manufacturer's instructions. One microgram of total RNA was reverse-transcribed using the FastKing RT Kit with gDNase (TIANGEN, Beijing, China). The qRT-PCR assay was conducted by a StepOnePlus ${ }^{\text {TM }}$ Real-Time PCR System (Applied Biosystems, USA). PCR assays were carried out using the SYBR ${ }^{\circledR}$ Premix ExTaq ${ }^{\mathrm{TM}}$ II $(2 \times)$ kit (Takara, Tokyo, Japan). The relative expression of mRNA was quantified via normalization to watermelon $\beta$-actin ${ }^{50}$, and calculated as described previously ${ }^{51}$. For the semiquantitative RT-PCR assay, PCR-amplified products were electrophoresed on 1\% TAE-agarose gels. The genespecific primers are shown in Supplementary Table S1.

\section{Melatonin assay}

Melatonin was extracted using the acetone-methanol method $^{52}$ and measured using an ELISA kit (Shanghai Lanpai Biotechnology Co., Ltd, Shanghai, China) according to the manufacturer's instructions. Colorimetric recording was carried out via a Multimode Plate Reader M200 pro (Tecan, Männedorf, Switzerland).

\section{Cold, drought, and salt treatment of watermelon}

Watermelon (cv. Nongkeda No. 5) seedlings were cultured in a greenhouse at Northwest Agriculture and Forestry University, Yangling, China. Seedlings with three true leaves were treated with cold at $4{ }^{\circ} \mathrm{C}$, unwatered, or irrigated with $\mathrm{NaCl}$ solution $(300 \mathrm{mM}, 80 \mathrm{~mL}$ per plant). Leaf samples were taken after cold, drought, or salt treatment for $24 \mathrm{~h}, 4 \mathrm{~d}$, or $2 \mathrm{~d}$, respectively.

\section{Statistical analysis}

All data were analyzed with SPSS software by using Student's $t$ test, and $P<0.05$ was considered to indicate statistical significance.

\section{Results \\ Bioinformatics analysis and expression profiles of the CIOMT genes in watermelon}

A total of 16 putative ClOMT genes were identified in watermelon using the HMM search program and were 
Table 1 O-methyltransferase genes in watermelon

\begin{tabular}{|c|c|c|c|}
\hline Gene Name & Gene ID & Chromosome location & Description \\
\hline CIOMTO1 & Cla97C10G188660 & Chr10: 4750672.4753331 & caffeic acid 3-O-methyltransferase-like \\
\hline CIOMTO2 & Cla97C10G188670 & Chr10: 4767376.4770041 & caffeic acid 3-O-methyltransferase-like \\
\hline СІОМТОЗ & Cla97C07G144540 & Chr07: $31874671 . .31875744$ & caffeic acid 3-O-methyltransferase 1 \\
\hline CIOMT04 & Cla97C02G043210 & Chr02: 31427713.31428863 & O-methyltransferase, family 2 \\
\hline CIOMT05 & Cla97C02G043200 & Chr02: 31402942.31404092 & O-methyltransferase, family 2 \\
\hline CIOMTO6 & Cla97C09G172390 & Chr09: $8789656 . .8791046$ & O-methyltransferase, family 2 \\
\hline СІОМТО7 & Cla97C02G028680 & Chr02: $2161556 . .2164053$ & O-methyltransferase, family 2 \\
\hline СІОМТО8 & Cla97C02G030510 & Chr02: 3482833.3484720 & O-methyltransferase, family 2 \\
\hline СІОМTO9 & Cla97C02G030520 & Chr02: 3488829.3491274 & O-methyltransferase, family 2 \\
\hline CIOMT10 & Cla97C02G030540 & Chr02: 3508413.3510435 & O-methyltransferase, family 2 \\
\hline CIOMT11 & Cla97C02G030550 & Chr02: 3515980.3518336 & O-methyltransferase, family 2 \\
\hline CIOMT12 & Cla97C02G030560 & Chr02: $3542077 . .3543859$ & O-methyltransferase, family 2 \\
\hline CIOMT13 & Cla97C02G030570 & Chr02: $3547673 . .3554475$ & O-methyltransferase, family 2 \\
\hline CIOMT14 & Cla97C10G195930 & Chr10: $25518757 . .25520719$ & O-methyltransferase \\
\hline CIOMT15 & Cla97C10G195920 & Chr10: $25509231 . .25511161$ & O-methyltransferase \\
\hline CIOMT16 & Cla97C10G202790 & Chr10: $32622705 . .32628072$ & O-methyltransferase \\
\hline
\end{tabular}

The gene description sourced from the CuGenDB (http://cucurbitgenomics.org/). CIOMT Citrullus lanatus O-methyltransferase

renamed ClOMT01 to ClOMT16 based on their gene ID in the watermelon reference genome (Table 1). Among ClOMT proteins, ClOMT03 was annotated as caffeic acid 3-O-methyltransferase 1 , while ClOMT01 and ClOMT02 were annotated as caffeic acid 3-O-methyltransferase-like.

The 16 ClOMT genes were unevenly distributed on chromosomes 02, 07, 09 and 10 in the watermelon genome (Fig. 1A). Some ClOMTs mapped to neighboring regions on the same chromosome. According to the defined criteria ${ }^{38,39}$, ClOMT01, ClOMT02, ClOMT03, ClOMT06, ClOMT07, and ClOMT16 were not tandemly duplicated genes, while the other ClOMT genes seemed to be produced from tandem duplications. As shown in Fig. 1B, ClOMTs were classified into three groups (I-III) according to phylogenetic analyses, with ten ClOMTs in group I, two in group II (ClOMT04 and ClOMT05), and four in group III (ClOMT01, ClOMT02, ClOMT03 and ClOMT16).

The temporal and spatial expression of $C l O M T$ genes was analyzed based on the RNA-seq data from CuGenDB (http://cucurbitgenomics.org/). Only ClOMTO2, ClOMT03 and ClOMT16 were constitutively expressed in all watermelon tissues tested, including fruit flesh, fruit rind, seed, phloem, vascular bundle, root, and leaf tissues (Fig. 2A). The ClOMT01 gene was undetected only in seeds, while ClOMTO4 and ClOMT05 were undetected only in fruit flesh 34 days after pollination. Most $\mathrm{ClOMT}$ genes in group
I were not detected in fruit flesh, fruit rind, or seeds. Expression of ClOMT14 was not observed in any of the tissues tested.

The production of melatonin can be induced by various abiotic stresses ${ }^{53}$. We further analyzed the expression of ClOMT genes in response to abiotic stresses, including cold, drought, and osmotic stress, simulated using 20\% PEG 6000. The ClOMT genes exhibited different expression patterns in response to different stresses (Fig. 2B). Only ClOMTO3 expression was upregulated by all three stresses. The expression of ClOMTO4 and ClOMT05 was upregulated under both drought and osmotic stress. The transcript levels of ClOMT08 and ClOMT16 were induced only by osmotic stress, while that of ClOMTO7 was induced only by drought.

\section{Sequence analysis and subcellular localization of the CICOMT1 protein}

To further narrow the range of candidate COMT genes, we analyzed the sequence identities of ClOMT01, ClOMT02, ClOMTO3 and ClOMT16 to the ASMTs and melatonin biosynthesis-related COMTs reported in other plant species (Table 2). These four ClOMTs had $<43 \%$ identity to ASMTs in Arabidopsis, Malus zumi, and rice. The ClOMT03 gene exhibited the highest identities to COMTs in Arabidopsis (74.93\%), tomato (74.30\%), and 
(A)

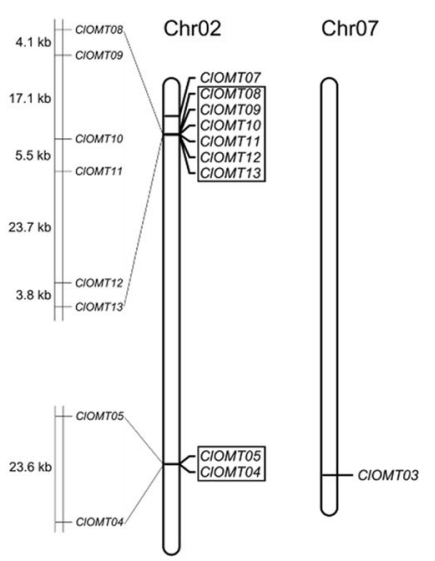

(B)

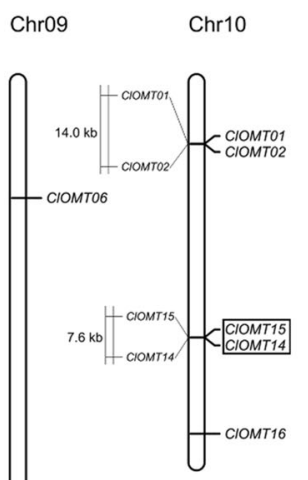

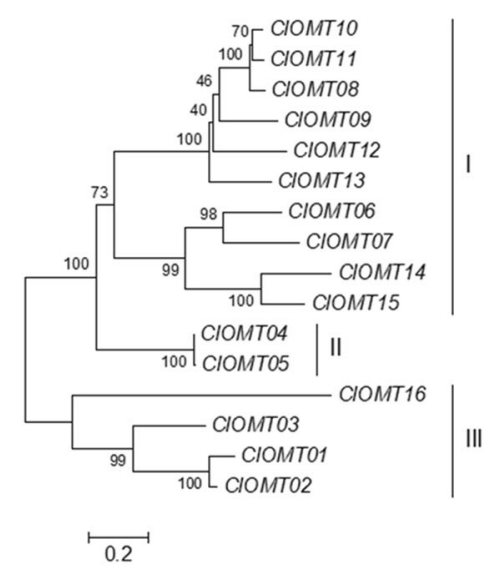

Fig. 1 Chromosomal locations and phylogenetic analysis of CIOMT genes in watermelon. A The chromosomal location was drawn using MapChart2.32. The tandem duplicated genes are boxed. B The phylogenetic tree of the CIOMT genes was constructed using MEGA 7.0.21, and the CIOMT genes were placed into groups I, II and III. Bar, 0.2 substitutions per site; Chr, chromosome; CIOMT Citrullus lanatus O-methyltransferase

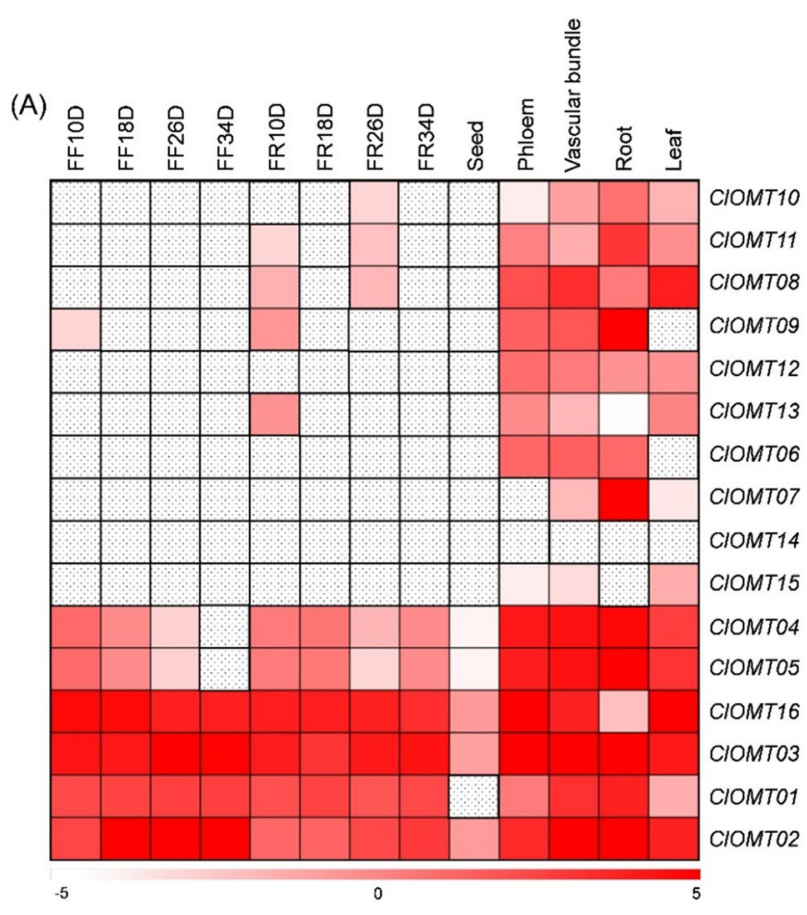

(B)

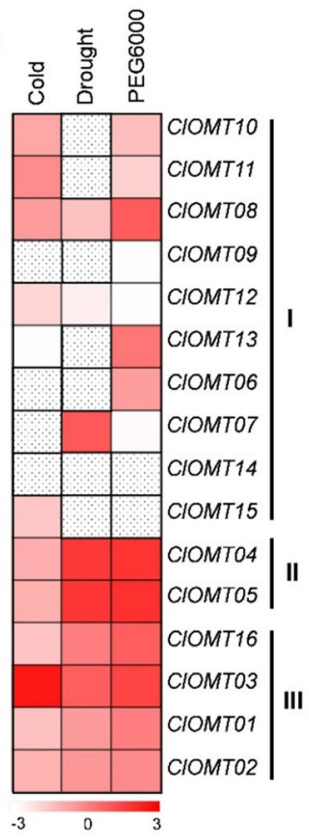

Fig. 2 The expression of CIOMT genes in different tissues and in response to abiotic stresses. Heat maps of CIOMT genes in (A) different tissues and (B) in response to cold, drought, or osmotic stress. The RNA-seq data were obtained from the CuGenDB (http://cucurbitgenomics.org/). Color coding was done according to the scale given. Boxes filled with dots indicate no detection. In (A), watermelon tissues included fruit flesh (FF), fruit rind (FR) 10, 18, 26, and 34 days after pollination, seeds 49 days after pollination, phloem, vascular bundle, root, and leaf tissues. Transcript levels were calculated as log2transformed Reads Per Kilobase of exon model per Million mapped reads (RPKM). In (B), the watermelon seedlings were exposed to $4^{\circ} \mathrm{C}$ for $6 \mathrm{~h}$, unwatered for eight days, or treated with PEG 6000 (20\%) on roots for 6 h. Leaf tissues were harvested after cold and drought, while root samples were taken after PEG 6000 treatment. The values were calculated as log2-transformed RPKM ratios of Treatment/Control. CIOMT Citrullus lanatus O-methyltransferase

rice (60.00\%). Based on the above results, ClOMT03 was considered a potential watermelon COMT gene involved in melatonin biosynthesis and was renamed ClCOMT1.
Like the known COMT proteins from dicotyledonous Arabidopsis and tomato, ClCOMT1 contained relatively conserved NAS-binding domains, phenolic substrate-binding 
Table 2 The homology of CIOMT01, CIOMT02, CIOMT03, and CIOMT16 to ASMT/COMT genes from other plant species

\begin{tabular}{|c|c|c|c|c|c|c|}
\hline \multirow[t]{2}{*}{ Gene Name } & \multicolumn{3}{|l|}{ COMT } & \multicolumn{3}{|l|}{ ASMT } \\
\hline & AtCOMT & SICOMT1 & OsCOMT & AtASMT & MzASMT1 & OsASMT1 \\
\hline CIOMT01 & $50.31 \%$ & $52.30 \%$ & $46.20 \%$ & $42.86 \%$ & $29.00 \%$ & $26.45 \%$ \\
\hline СІОМТ02 & $54.52 \%$ & $56.73 \%$ & $50.68 \%$ & $34.12 \%$ & $33.53 \%$ & $30.59 \%$ \\
\hline СІОМТОЗ & $74.93 \%$ & $74.30 \%$ & $60.00 \%$ & $33.13 \%$ & $32.09 \%$ & $28.21 \%$ \\
\hline CIOMT16 & $36.48 \%$ & $34.03 \%$ & $32.63 \%$ & $28.16 \%$ & $26.24 \%$ & $28.37 \%$ \\
\hline
\end{tabular}

AtCOMT, AT5G54160; SICOMT1, Solyc03g080180; OsCOMT, LOC_Os08g06100; AtASMT, AT4G35160; MzASMT1, MD05G1308800; OsASMT1, LOC_Os09g17560

At Arabidopsis thaliana, CI Citrullus lanatus, Mz Malus zumi, Os Oryza sativa, SI Solanum lycopersicum, ASMT N-acetylserotonin O-methyltransferase, COMT caffeic acid O-methyltransferase, OMT O-methyltransferase

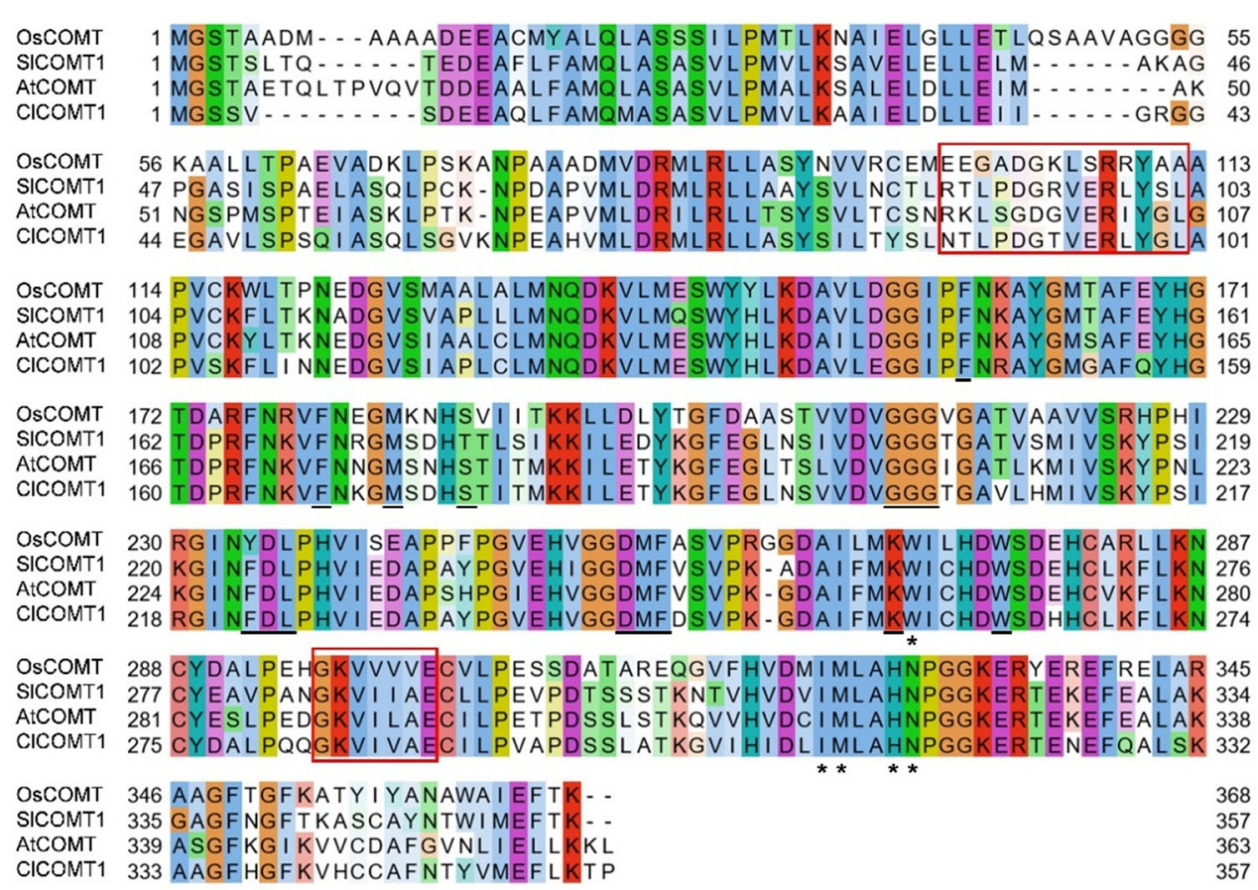

Fig. 3 Comparison of the CICOMT1 protein sequence with those of AtCOMT, SICOMT1, and OsCOMT. The putative N-acetylserotonin (NAS)binding domains are shown in the red box. The phenolic substrate-binding sites are marked with asterisks. The S-adenosyl-L-methionine (SAM)-binding sites are underlined. At Arabidopsis thaliana, Cl Citrullus lanatus, Os Oryza sativa, SI Solanum lycopersicum, COMT caffeic acid O-methyltransferase

residues, and $S$-adenosyl-L-methionine-binding sites (Fig. 3). However, the NAS-binding domains varied considerably between COMT proteins from dicotyledonous watermelon and monocotyledonous rice. The COMT proteins in rice and tomato are known to be localized in the cytoplasm ${ }^{21,30}$. To determine whether ClCOMT1 is also localized in the cytoplasm, the subcellular localization of ClCOMT1 in watermelon was investigated using the pGREEN-ClCOMT1-GFP vector. As shown in Fig. 4, green fluorescence was observed in the cytoplasm, indicating the cytoplasmic localization of ClCOMT1. The green fluorescence did not overlap with the red fluorescence of chlorophyll autofluorescence, suggesting that ClCOMT1 was not translocated into chloroplasts.

\section{Effects of CICOMT1 gene editing or overexpression on endogenous melatonin contents in watermelon calli}

To ascertain the direct involvement of ClCOMT1 in melatonin biosynthesis, transgenic watermelon calli with either knockout or overexpression of ClCOMT1 were generated. Knockout of ClCOMT1 in watermelon calli was performed using the CRISPR/Cas9 system. Two target sites on the 5'-region of the ClCOMT1 gene (target 1 and target 2) were assembled into the CRISPR/Cas9 vector PBSE402 


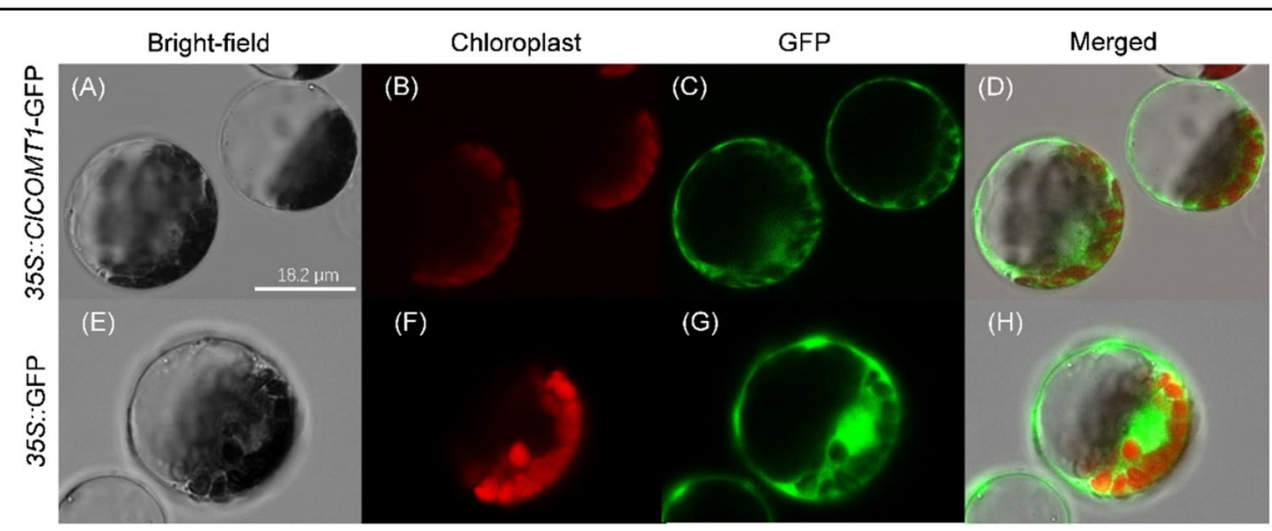

Fig. 4 Subcellular localization of CICOMT1 in watermelon protoplasts. A, E Bright-field image of watermelon protoplasts. B, F Red fluorescence of chlorophyll autofluorescence. C, G Green fluorescence of GFP. D, H Merged view of two fluorescence images $(B+C, F+G)$. Bars $=18.2 \mu m$. CICOMT Citrullus lanatus caffeic acid O-methyltransferase, GFP green fluorescent protein

(A)

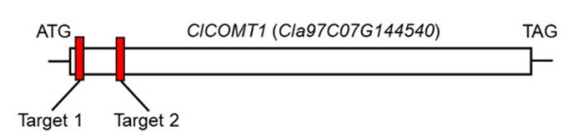

(B)

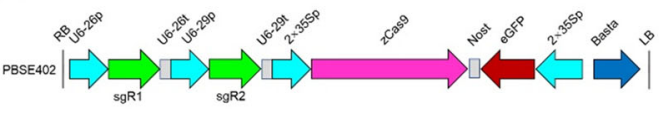

(C)

WT

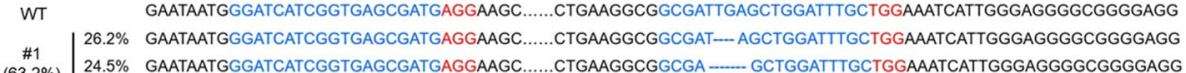

\begin{tabular}{c|ccc}
$\# 1$ & $24.5 \%$ & GAATAATGGGATCATCGGTGAGCGATGAGGAAGC........CTGAAGGCGGCGA -..... GCTGGATTTGCTGGAAATCATTGGGAGGGGCGGGGAGG \\
$(63.2 \%)$ & $12.5 \%$ & GAATAATGGGATCATCGGTGAGCGATGAGGAAGC.......CTGAAGGCG_... AGCTGGATTTGCTGGAAATCATTGGGAGGGGCGGGAGG
\end{tabular}

47.5\% GAATAATGGGATCATCGGTGAGCGATGAGGAAGC........ TTGAAGG...

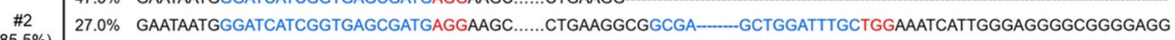

(85.5\%) $11.0 \%$ GAATAATGGGATCATCGGTGAGCGATGAGGAAGC.......... TTGAAGGCGGC …-... AGCTGGATTTGCTGGAAATCATTGGGAGGGGCGGGGAGG

30.1\% GAATAATGGGATCATCGGTGAGCGATGAGGAAGC.......CTGAAGGCGGCGAT -- AGCTGGATTTGCTGGAAATCATTGGGAGGGGCGGGGAGG

\begin{tabular}{c|ccc}
$\# 3$ & $28.2 \%$ & GAATAATGGGATCATCGGTGAGCGATGAGGAAGC........CTGAAGGCGGCGA \\
$(79.7 \%)$ & $11.0 \%$ & GAATAATGGGATCATCGGTGAGCGATGAGGAAGC........CTGAAGGCGGCGA
\end{tabular}

$10.4 \%$ GAATAATGGGATCATCGGTGAGCGATGAGGAAGC.........TTGAAGGCGGCGA

\#4 $\mid 37.2 \%$ GAATAATGGGATCATCGGTGAGCGATGAGGAAGC........ TTGAAGGCGG $\ldots$

(74.0\%) $36.8 \%$ GAATAATGGGATCATCGGTGAGCGATGAGGAAGC........CTGAAGGCGGCGA-

\#5 $\mid 54.2 \%$ GAATAATG -...-AGGAAGC........ CTGAAGGCGGCGATTGAGCTGGATTTGCTGGAAATCATTGGGAGGGGGGGGAGG

(100\%) $45.8 \%$ GAATAATGGGATCA _.................. ATGAGGAAGGCGGCGATTGAGCTGGATTTGCTGGAAATCATTGGGAGGGGCGGGGAGG

Fig. 5 Effects of CICOMT1 gene-editing on endogenous melatonin contents in watermelon calli. A Two sgRNAs (Target 1 and Target 2) in the 5'-region of CICOMT1. B Schematic diagram for constructing two sgRNA cassettes in the binary vector PBSE402. C Mutation types and rates in the transgenic watermelon calli after CRISPR/Cas9-mediated gene editing. D Melatonin contents in transgenic and wild-type (WT) calli. In (D), values are means \pm SD $(n=3)$. Asterisk $\left(^{*}\right)$ indicates significant difference at $P<0.05$. CICOMT Citrullus lanatus caffeic acid O-methyltransferase

(Fig. 5A, B). After transformation, five edited lines were obtained. Four lines (\#1-\#4) had mutations in the target 2 site, and one line (\#5) had mutations in the target 1 site (Fig. $5 \mathrm{C})$. Line $\# 5$ had the highest mutation rate (100\%), followed by \#2 (85.5\%), \#3 (79.7\%), \#4 (74.0\%), and \#1 (63.2\%). All lines were heterozygous with 2-4 mutation types for each. For example, \#5 had two mutation types $(-19,-10)$ in target 1 , whereas \#3 had four $(-2,-35$,
$-24,-36)$ in target 2. Mutation of ClCOMT1 resulted in a significant decrease in melatonin content (Fig. 5D). Melatonin contents in edited lines were 33.7-56.3\% lower than those in wild-type calli. Moreover, melatonin content was negatively correlated with the mutation rate (Fig. S1).

In addition, $C l C O M T 1$ driven by the $35 \mathrm{~S}$ promoter was genetically transformed into watermelon calli, leading to 


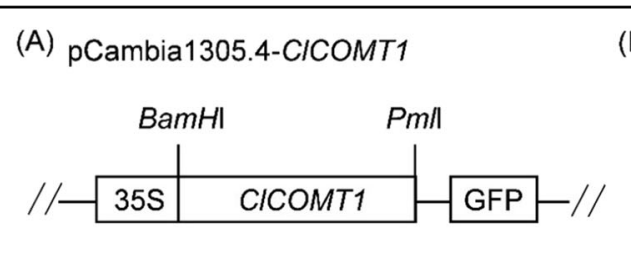

(B)

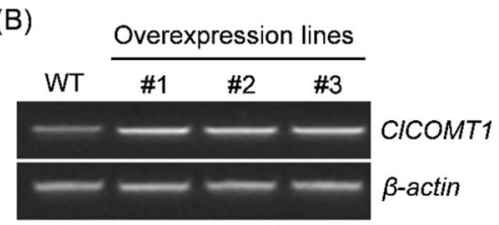

(C)

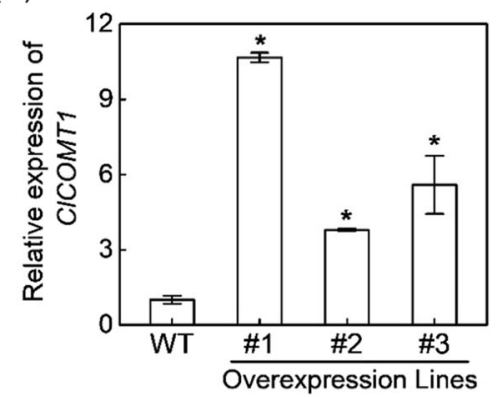

(D)

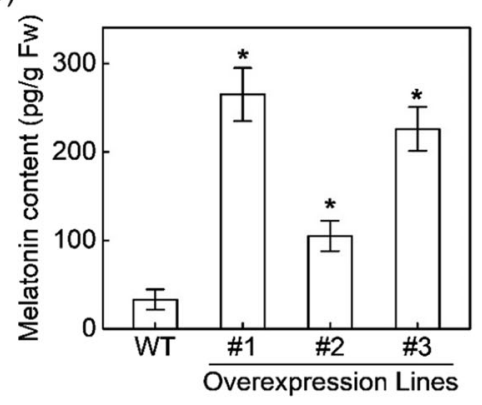

Fig. 6 Effects of CICOMT1 overexpression on endogenous melatonin contents in watermelon calli. A Schematic diagram of the pCambia1305.4CICOMT1 vector. B, C RT-PCR and qRT-PCR analysis of CICOMT1 transcripts in overexpression lines and wild-type (WT) calli. D Melatonin contents in overexpression lines and WT calli. $\beta$-actin, watermelon internal reference gene. In $(\mathbf{C}, \mathbf{D})$, values are means \pm SD $(n=3)$. Asterisk $(*)$ indicates significant difference at $P<0.05$. CICOMT Citrullus lanatus caffeic acid O-methyltransferase

the overexpression of ClCOMT1 (Fig. 6A-C). The transcript levels of ClCOMT1 in transgenic lines \#1, \#2, and \#3 were induced by $10.7-, 3.8-$, and 5.6-fold relative to those in nontransgenic calli, respectively. Consequently, the contents of melatonin in $\# 1, \# 2$, and \#3 increased by 7.0-, 2.2-, and 5.8-fold compared to that in wild-type calli (Fig. 6D). These results indicated a direct role of ClCOMT1 in melatonin production in watermelon.

\section{The involvement of CICOMT1 in abiotic stresses}

The application of exogenous melatonin enhanced watermelon tolerance against cold, drought, and salt stress, as assessed through the plant phenotypes and relative electrical conductivity (Fig. S2). After exposure to these three stresses, ClCOMT1 transcripts were induced by 2.1-, 4.7-, and 7.0-fold, and the melatonin contents were increased by $82.1 \%, 41.7 \%$, and $125.9 \%$, respectively (Fig. 7).

To further examine whether ClCOMT1 conferred plant tolerance to abiotic stresses, this gene was genetically transformed into Arabidopsis using a pGREENClCOMT1-GFP vector (Fig. 8A). The expression of ClCOMT1 was detected in the transgenic plants but not in wild-type plants (Fig. 8B). Melatonin contents in five transgenic lines were increased by 2.3- to 4.1-fold compared to that in the wild plants (Fig. 8C). Transgenic plants exhibited higher tolerance to cold, osmotic stress, and $\mathrm{NaCl}$ (Fig. 8D, E). For instance, the survival rates of transgenic plants (line \#4) were $77.5 \%, 82.2 \%$, and $79.6 \%$, far higher than $31.3 \%, 53.9 \%$, and $53.8 \%$ in wild plants after cold, mannitol, and $\mathrm{NaCl}$ treatment, respectively.
These results indicated that ClCOMT1 is a positive regulator of plant tolerance to abiotic stresses.

\section{Discussion \\ CIOMT03 (Cla97C07G144540) is a potential watermelon COMT gene (CICOMT1)}

The final step in melatonin biosynthesis is the conversion of NAS by proteins with ASMT activity. At present, ASMT genes have been cloned from rice, Malus zumi, Arabidopsis, and Hypericum perforatum ${ }^{15,17-19}$. However, ASMT genes are not conserved and thus are difficult to clone due to the lack of candidate genes in other plant species ${ }^{15-17}$, especially in those in which transformation is difficult, such as Cucurbitaceae. An alternative enzyme, COMT, also has ASMT activity and can methylate NAS into melatonin ${ }^{20}$. Surprisingly, the relative ASMT activity $\left(V_{\max } / K_{m}\right)$ of AtCOMT in melatonin synthesis was far higher than that of AtASMT both in vivo in Arabidopsis and in vitro ${ }^{20}$. In contrast, the substrate affinity $\left(K_{\mathrm{m}}\right)$ of ASMT for NAS was higher than that of COMT, suggesting higher NAS affinity for $\mathrm{COMT}^{20}$. In addition to NAS, serotonin was also found to be catalyzed by COMT into 5-methoxytryptamine, which was converted into melatonin by $\mathrm{SNAT}^{54}$.

As COMTs belong to the OMT family, 16 putative ClOMT genes with an O-methyltransferase domain (PF00891) were identified in watermelon (Table 1$)^{22,55}$. It is widely reported that melatonin exists in almost all plant tissues and that its production can be induced by various abiotic stresses, such as cold, drought, salinity, and excess heavy metals ${ }^{6,53,56}$. According to these previous results, 

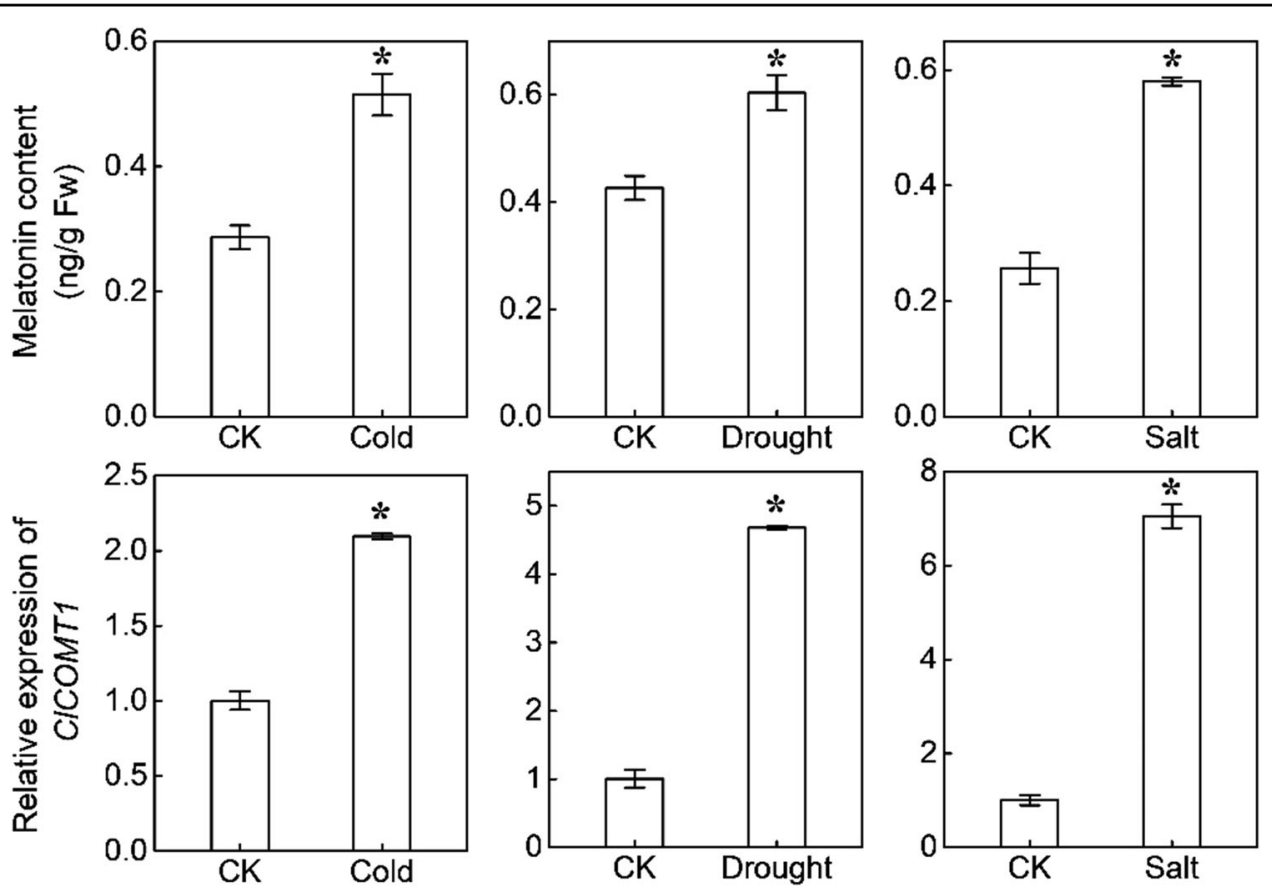

Fig. 7 Melatonin contents and CICOMT1 expression in response to cold, drought, or salt stress. Watermelon seedlings with three true leaves were exposed to cold at $4{ }^{\circ} \mathrm{C}$, unwatered, or irrigated with $300 \mathrm{mM} \mathrm{NaCl}(80 \mathrm{~mL}$ per plant). Leaf samples were taken after cold, drought, or salt treatment for $24 \mathrm{~h}, 4 \mathrm{~d}$, or $2 \mathrm{~d}$, respectively. Values are means $\pm \mathrm{SD}(n=3)$. An asterisk $\left.{ }^{*}\right)$ indicates significant difference at $P<0.05$. CK control check, CICOMT, Citrullus lanatus caffeic acid O-methyltransferase

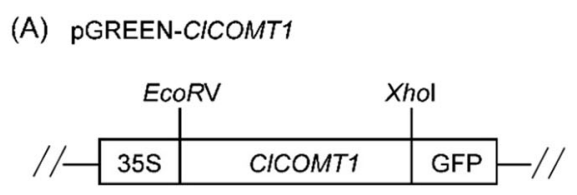

(B)

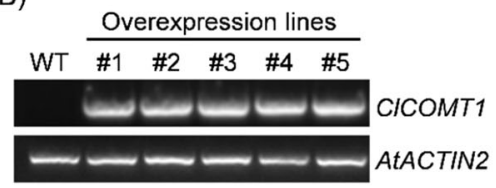

(D)

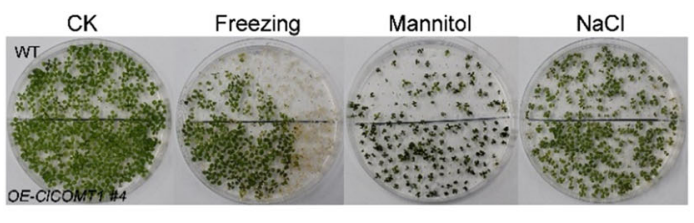

(C)

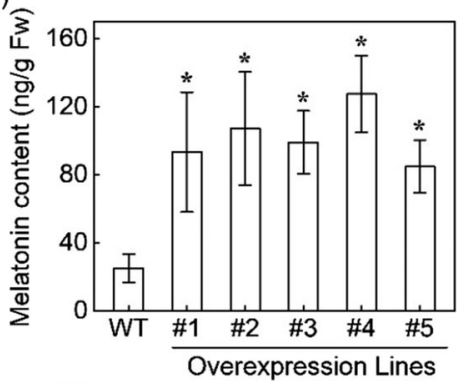

(E)

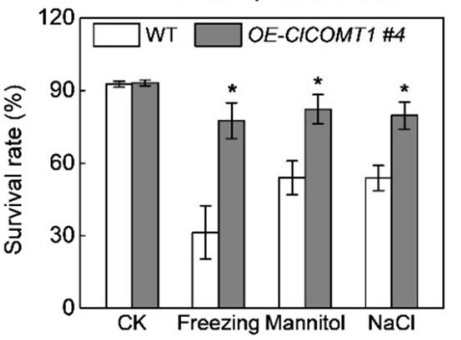

Fig. 8 Effects of CICOMT1 overexpression on melatonin contents and abiotic stress tolerance in Arabidopsis. A Schematic diagram of the PGREEN-CICOMT1 vector. B RT-PCR analysis of CICOMT1 expression in transgenic and wild-type (WT) Arabidopsis. C Melatonin contents in transgenic and WT Arabidopsis. D, E Phenotypes and survival rates of transgenic (line \#4) and WT Arabidopsis after freezing, mannitol, or NaCl treatment. For freezing treatment, two-week-old Arabidopsis seedlings were exposed to $-10^{\circ} \mathrm{C}$ for $1 \mathrm{~h}$, followed by recovery at $22^{\circ} \mathrm{C}$ for $4 \mathrm{~d}$. For drought and salt stress, Arabidopsis seeds were sown and grown on $1 / 2 \mathrm{MS}$ containing $250 \mathrm{mM}$ D-Mannitol and $75 \mathrm{mM} \mathrm{NaCl}$, respectively. In (C, E), values are means \pm SD $(n=3)$. Asterisk $\left(^{*}\right)$ indicates significant difference at $P<0.05$. CK, control check; CICOMT, Citrullus lanatus caffeic acid O-methyltransferase 
bioinformatics analysis of $C l O M T \mathrm{~s}$ was performed to screen candidate ClCOMT genes. ClOMT03 (Cla97C07G144540) was considered a potential ClCOMT gene (renamed ClCOMT1) based on its high identities (60.00-74.93\%) to known COMT genes involved in melatonin biosynthesis, constitutive expression in all tissues, and upregulation under abiotic stresses (Figs. 1, 2). In addition to the known COMT proteins $^{20,21}$, ClCOMT1 was localized in the cytoplasm, where melatonin is synthesized from $\mathrm{NAS}^{54}$. In addition, the amino acid sequences and NAS-binding domains were relatively conserved among COMTs from dicotyledonous plants but not between COMTs from dicotyledonous and monocotyledonous species, suggesting that the COMT genes from dicotyledonous plants may originate from the same lineage (Fig. 3).

\section{CICOMT1 plays a direct role in melatonin biosynthesis in watermelon}

Overexpression or knockout of ClCOMT1 significantly increased or decreased melatonin contents, respectively, in the transgenic watermelon calli (Figs. 5, 6). Moreover, the melatonin contents were negatively correlated with the mutation rates of ClCOMT1 in mutant watermelon calli (Fig. S1). These results indicated that ClCOMT1 plays an essential role in melatonin synthesis in watermelon. In previous studies, COMT overexpression induced a slight increase in melatonin content in transgenic plants ${ }^{21,30,57}$. For instance, the SlCOMT1 transcripts increased by 9 -fold in transgenic tomato, whereas the melatonin content increased only by $35.2 \%$ compared to that in the nontransformed plants $^{57}$. Interestingly, in watermelon calli with ClCOMT1 overexpression, the increased levels of ClCOMT1 expression (3.8- to 10.7-fold) were closely coupled with increases in melatonin contents, which were 2.2- to 7.0-fold relative to those in wild calli (Fig. 6). The difference in results may be attributed to the use of different tissues or species.

COMT is a multifunctional enzyme catalyzing the methylation of a diverse set of substrates such as 5-hydroxyferulic acid, caffeic acid, and quercetin ${ }^{58}$. The melatonin synthesis activity of COMT via NAS methylation is highly inhibited by the aforementioned substrates ${ }^{20,21,59}$. Owing to the ubiquitous presence of substrates that act as bottlenecks in the production of melatonin, ASMTs are required in plant species $^{21}$. Here, we observed that transgenic watermelon calli with $100 \%$ mutation of ClCOMT1 had $43.7 \%$ melatonin compared to wild calli (Fig. 5D). Therefore, it is plausible that there are ClASMT or other ClCOMT genes contributing to melatonin biosynthesis in watermelon. However, this speculation requires further investigation.

\section{CICOMT1 is a positive regulator of plant tolerance to abiotic stresses}

Increasing studies have demonstrated that melatonin functions as an important regulator in plant adaptation to various environmental stresses. Application of melatonin at appropriate concentrations can enhance watermelon tolerance against cold, drought, and $\mathrm{NaCl}^{31-33}$. SlCOMT1 overexpression enhanced salt tolerance in transgenic tomato plants ${ }^{30,57}$. Here, the expression of ClCOMT1 was clearly induced, accompanied by increases in the accumulation of melatonin, after plants were challenged with cold, drought, and $\mathrm{NaCl}$ stress (Fig. 7). Furthermore, transgenic Arabidopsis plants with ClCOMT1 expression showed higher tolerance to freezing, mannitol, and $\mathrm{NaCl}$ than the wild plants (Fig. 8). Taken together, ClCOMT1 plays a positive role in regulating plant tolerance against abiotic stresses, and this role of ClCOMT1 might be related to increased melatonin accumulation. In addition, COMT can methylate phenylpropanoid and flavonoid substrates and plays a key role in lignin biosynthesis ${ }^{23}$. In addition to melatonin, phenylpropanoids, flavonoids, and lignin play important roles in plant defense against various environmental stresses ${ }^{60-63}$. Therefore, ClCOMT1induced abiotic stress tolerance might also be associated with altered phenylpropanoids, flavonoids, or lignin, but such speculation needs to be further investigated.

\section{Conclusion}

As more functions of melatonin in plants have been confirmed, the key genes involved in melatonin synthesis should be explored in more plant species. In the current study, we reported a watermelon COMT gene (ClCOMT1) that plays an essential role in melatonin biosynthesis. We also confirmed that ClCOMT1 is a positive regulator of plant tolerance to abiotic stresses, including cold, drought, and salinity. To our knowledge, ClCOMT1 is the first melatonin biosynthetic gene cloned from a species in the Cucurbitaceae. It is likely that the findings presented here could be exploited to benefit cucurbit crop production in favorable and unfavorable environmental conditions.

\section{Acknowledgements \\ This study was supported by the National Key Research and Development Program of China (2018YFD1000800), the National Natural Science Foundation of China $(31972479,31801884)$, the Earmarked Fund for Modern Agroindustry Technology Research System of China (CARS-25), and the funding for Tang Scholar of Northwest A\&F University.}

\section{Author contributions}

$\mathrm{HL}, X Z$, and JC planned and designed the research; JC, HL, YG, JY, LY, ZZ, CW, $Y Z, J M$ and JQY performed experiments and analyzed the data; JC, HL, and LY wrote and revised the paper.

\section{Competing interests}

The authors declare no competing interests.

Supplementary information The online version contains supplementary material available at https://doi.org/10.1038/s41438-021-00645-5. 
Received: 31 January 2021 Revised: 18 May 2021 Accepted: 22 June 2021 Published online: 01 October 2021

\section{References}

1. Hardeland, R., Cardinali, D. P. \& Srinivasan, V. Melatonin-A pleiotropic, orchestrating regulator molecule. Prog. Neurobiol. 93, 350-384 (2011).

2. Arnao, M. B. Phytomelatonin: discovery, content, and role in plants. Adv. Bot. 2014, e815769 (2014).

3. Dubbels, R. et al. Melatonin in edible plants identified by radioimmunoassay and by high performance liquid chromatography-mass spectrometry. J. Pineal Res. 18, 28-31 (1995).

4. Hattori, A., Migitaka, H., ligo, M., Itoh, M. \& Reiter, R. J. Identification of melatonin in plants and its effects on plasma melatonin levels and binding to melatonin receptors in vertebrates. Biochem. Mol. Biol. Int. 35, 627-634 (1995).

5. Arnao, M. B. \& Hernandez-Ruiz, J. Melatonin: plant growth regulator and/or biostimulator during stress? Trends Plant Sci. 19, 789-797 (2014).

6. Zhang, N. et al. Roles of melatonin in abiotic stress resistance in plants. J. Exp. Bot. 66, 647-656 (2014).

7. Sun, Y. D. et al. Melatonin treatment improves the shelf-life and postharvest quality of table grape (Vitis labrusca L. cv. 'Fengzao'). J. Berry Res. 10, 1-11 (2020).

8. Wei, J. et al. Phytomelatonin receptor PMTR1-mediated signaling regulates stomatal closure in Arabidopsis thaliana. J. Pineal Res. 65, e12500 (2018).

9. Arnao, M. B. \& Hernández-Ruiz, J. Melatonin: a new plant hormone and/or a plant master regulator? Trends Plant Sci. 24, 38-48 (2018).

10. Tan, D. X. et al. Functional roles of melatonin in plants, and perspectives in nutritional and agricultural science. J. Exp. Bot. 63, 577-597 (2012).

11. Park, W. J. Melatonin as an endogenous plant regulatory signal-debates and perspectives. J. Plant Biol. 54, 143-149 (2011).

12. Back, K., Tan, D. X. \& Reiter, R. J. Melatonin biosynthesis in plants: multiple pathways catalyze tryptophan to melatonin in the cytoplasm or chloroplasts. J. Pineal Res. 61, 426-437 (2016).

13. Byeon, Y., Lee, H. Y., Lee, K., Park, S. \& Back, K. Cellular localization and kinetics of the rice melatonin biosynthetic enzymes SNAT and ASMT. J. Pineal Res. 56, 107-114 (2014).

14. Kang, K., Lee, K., Park, S., Byeon, Y. \& Back, K. Molecular cloning of rice serotonin $\mathrm{N}$-acetyltransferase, the penultimate gene in plant melatonin biosynthesis. J. Pineal Res. 55, 7-13 (2013).

15. Kang, K. et al. Molecular cloning of a plant $N$-acetylserotonin methyltransferase and its expression characteristics in rice. J. Pineal Res. 50, 304-309 (2011).

16. Park, S., Byeon, Y. \& Back, K. Functional analyses of three ASMT gene family members in rice plants. J. Pineal Res. 55, 409-415 (2013).

17. Byeon, Y., Lee, H. J., Lee, H. Y. \& Back, K. Cloning and functional characterization of the Arabidopsis $\mathrm{N}$-acetylserotonin O-methyltransferase responsible for melatonin synthesis. J. Pineal Res. 60, 65-73 (2016).

18. Zuo, B. et al. Overexpression of MzASMT improves melatonin production and enhances drought tolerance in transgenic Arabidopsis thaliana plants. J. Pineal Res. 57, 408-417 (2014).

19. Zhou, W. et al. Whole-genome sequence data of Hypericum perforatum and functional characterization of melatonin biosynthesis by $\mathrm{N}$-acetylserotonin $\mathrm{O}$ methyltransferase. J. Pineal Res. 00, e12709 (2020).

20. Byeon, Y., Lee, H. Y., Lee, K. \& Back, K. Caffeic acid O-methyltransferase is involved in the synthesis of melatonin by methylating $\mathrm{N}$-acetylserotonin in Arabidopsis. J. Pineal Res. 57, 219-227 (2014).

21. Byeon, Y., Choi, G. H., Lee, H. Y. \& Back, K. Melatonin biosynthesis requires Nacetylserotonin methyltransferase activity of caffeic acid O-methyltransferase in rice. J. Exp. Bot. 66, 6917-6925 (2015).

22. Lam, K. C., Ibrahim, R. K., Behdad, B. \& Dayanandan, S. Structure, function, and evolution of plant O-methyltransferases. Genome 50, 1001-1013 (2007).

23. Tu, Y. et al. Functional analyses of caffeic acid O-methyltransferase and cinnamoyl-CoA-reductase genes from perennial ryegrass (Lolium perenne). Plant Cell 22, 3357-3373 (2010).

24. Louie, G. V. et al. Structure-function analyses of a caffeic acid O-methyltransferase from perennial nyegrass reveal the molecular basis for substrate preference. Plant Cell 22, 4114-4127 (2010).

25. LV, G. et al. The anatomy and physiology of spray cut chrysanthemum pedicels, and expression of a caffeic acid 3-O-methyltransferase homologue. Postharvest Biol. Tec. 60, 244-250 (2011).
26. Daly, P. et al. RNAi-suppression of barley caffeic acid O-methyltransferase modifies lignin despite redundancy in the gene family. Plant Biotechnol. J. 17, 594-607 (2019).

27. Trabucco, G. M. et al. Functional characterization of cinnamyl alcohol dehydrogenase and caffeic acid O-methyltransferase in Brachypodium distachyon. BMC Biotechnol. 13, 61 (2013).

28. Wu, X. et al. Phylogenetic, molecular, and biochemical characterization of caffeic acid O-methyltransferase gene family in Brachypodium distachyon. Int. J. Plant Genomics. 2013, 423189 (2013).

29. Meng, H. \& Campbell, W. Substrate profiles and expression of caffeoyl coenzyme A and caffeic acid O-methyltransferases in secondary xylem of aspen during seasonal development. Plant Mol. Biol. 38, 513-520 (1998).

30. Liu, D. D. et al. Overexpression of the melatonin synthesis-related gene SICOMT1 improves the resistance of tomato to salt stress. Molecules 24, 1514 (2019).

31. Li, H. et al. Local melatonin application induces cold tolerance in distant organs of Citrullus lanatus L. via long distance transport. Sci. Rep. 7, 40858 (2017).

32. $\mathrm{Li}, \mathrm{H}$. et al. Exogenous melatonin confers salt stress tolerance to watermelon by improving photosynthesis and redox homeostasis. Front Plant Sci. 8, 295 (2017).

33. Li, H. et al. Alkanes (C29 and C31)-mediated intracuticular wax accumulation contributes to melatonin- and ABA-induced drought tolerance in watermelon. J. Plant Growth Regul. 39, 1441-1450 (2020).

34. Nawaz, M. A. et al. Melatonin pretreatment improves vanadium stress tolerance of watermelon seedlings by reducing vanadium concentration in the leaves and regulating melatonin biosynthesis and antioxidant-related gene expression. J. Plant Physiol. 220, 115-127 (2018).

35. Guo, S. et al. Resequencing of 414 cultivated and wild watermelon accessions identifies selection for fruit quality traits. Nat. Genet. 51, 1616-1623 (2019).

36. Letunic, I., Khedkar, S. \& Bork, P. 20 years of the SMART protein domain annotation resource. Nucleic Acids Res. 46, 493-496 (2017).

37. Voorrips, R. E. MapChart: software for the graphical presentation of linkage maps and QTLs. J. Hered. 93, 77-78 (2002).

38. Zhu, Y. et al. Soybean (Glycine max) expansin gene superfamily origins: segmental and tandem duplication events followed by divergent selection among subfamilies. BMC Plant Biol. 14, 93 (2014).

39. Zhao, P. et al. Genome-wide analysis of the potato Hsp2O gene family: identification, genomic organization and expression profiles in response to heat stress. BMC Genomics 19, 61 (2018).

40. Larkin, M. A., Blackshields, G., Brown, N. P., Chenna, R. M. \& Higgins, D. G. ClustalW and ClustalX version 2. Bioinformatics 23, 2947-2948 (2007).

41. Kumar, S., Stecher, G. \& Tamura, K. MEGA7: molecular evolutionary genetics analysis version 7.0 for bigger datasets. Mol. Biol. Evolut. 33, 1870-1874 (2016).

42. Saeed, A. I. et al. TM4: a free, open-source system for microarray data management and analysis. Biotechniques 34, 374-378 (2003).

43. Tian, S. et al. Efficient CRISPR/Cas9-based gene knockout in watermelon. Plant Cell Rep. 36, 399-406 (2016).

44. Xing, H. L. et al. A CRISPR/Cas9 toolkit for multiplex genome editing in plants. BMC Plant Biol. 14, 327 (2014).

45. Liu, H. et al. CRISPR-P 2.0: an improved CRISPR/Cas9 tool for genome editing in plants. Mol. Plant 10, 530-532 (2017)

46. Liu, Q. et al. Hi-TOM: a platform for high-throughput tracking of mutations induced by CRISPR/Cas systems. Sci. China Life Sci. 62, 1-7 (2019).

47. Clough, S. J. \& Bent, A. F. Floral dip: a simplified method for Agrobacterium mediated transformation of Arabidopsis thaliana. Plant J. 16, 735-743 (1998).

48. Hu, Y., Jiang, L., Wang, F. \& Yu, D. Jasmonate regulates the inducer of CBF expression-C-repeat binding factor/DRE binding factor1 cascade and freezing tolerance in. Arabidopsis. Plant Cell. 25, 2907-2924 (2013).

49. Huang, $Y$. et al. Tissue-specific respiratory burst oxidase homolog-dependent $\mathrm{H}_{2} \mathrm{O}_{2}$ signaling to the plasma membrane $\mathrm{H}^{+}$-ATPase confers potassium uptake and salinity tolerance in. Cucurbitaceae. J. Exp. Bot. 70, 5879-5893 (2019).

50. Kong, Q. S. et al. Identification of suitable reference genes for gene expression normalization in qRT-PCR analysis in watermelon. PloS ONE 9, e90612 (2014).

51. Livak, K. J. \& Schmittgen, T. D. Analysis of relative gene expression data using real-time quantitative $P C R$ and the $2^{-\Delta \triangle C T}$ method. Methods 25, 402-408 (2001).

52. Pape, C. \& Lüning, K. Quantification of melatonin in phototrophic organisms. J. Pineal Res. 41, 157-165 (2006). 
53. Altaf, M. A. et al. Phytomelatonin: an overview of the importance and mediating functions of melatonin against environmental stresses. Physiol Plant. 172, 1-27 (2020).

54. Lee, H. Y., Byeon, Y., Lee, K., Lee, H. J. \& Back, K. Cloning of Arabidopsis serotonin $\mathrm{N}$-acetyltransferase and its role with caffeic acid O-methyltransferase in the biosynthesis of melatonin in vitro despite their different subcellular localization. J. Pineal Res. 75, 418-426 (2014).

55. Keller, N. P., Dischinger, H. C., Bhatnagar, D., Cleveland, T. E. \& Ullah, A. H. Purification of a 40-kilodalton methyltransferase active in the aflatoxin biosynthetic pathway. Appl Environ. Microbiol. 59, 479-484 (1993).

56. Gong, B. et al. Hydrogen peroxide produced by NADPH oxidase: a novel downstream signaling pathway in melatonin-induced stress tolerance in Solanum lycopersicum. Physiol. Plant. 160, 396-409 (2017).

57. Sun, S. et al. Overexpression of caffeic acid O-methyltransferase 1 (COMT1) increases melatonin level and salt stress tolerance in tomato plant. J. Plant Growth Regul. 39, 1221-1235 (2019).
58. Nakatsubo, T. et al. At5g54160 gene encodes Arabidopsis thaliana 5-hydroxyconiferaldehyde O-methyltransferase. J. Wood Sci. 54, 312-317 (2008).

59. Koshiba, T. et al. Characterization of 5-hydroxyconiferaldehyde O-methyltransferase in Oryza sativa. Plant Biotechnol. 30, 157-167 (2013).

60. Solecka, D. Role of phenylpropanoid compounds in plant responses to different stress factors. Acta Physiol. Plant 19, 257-268 (1997).

61. Fini, A., Brunetti, C., Ferdinando, M. D., Ferrini, F. \& Tattini, M. Stress-induced flavonoid biosynthesis and the antioxidant machinery of plants. Plant Signal Behav. 6, 709-711 (2011).

62. Baxter, H. L. \& Stewart Jr, C. N. Effects of altered lignin biosynthesis on phenylpropanoid metabolism and plant stress. Biofuels 4, 635-650 (2013).

63. Wen, W., Wang, R., Su, L., Lv, A. \& An, Y. MsWRKY11, activated by MsWRKY22, functions in drought tolerance and modulates lignin biosynthesis in alfalfa (Medicago sativa L.). Environ. Exp. Bot. 184, 104373 (2021). 